\title{
LA EVALUACIÓN INVESTIGATIVA EN CIENCIAS A NIVEL UNIVERSITARIO
}

Guido Alfredo Moncayo * Francia María Cabrera

\begin{abstract}
The evaluation plays different roles in the educational process. It should be considered as a learning process and not as a result of learning. In this paper we develop the strategy of evaluation as a research process, in which the student has the opportunity to summarize to solve problems, to read papers to integrate knowledge to develop competence in which he must apply the knowledge and socialize his own research.
\end{abstract}

\section{PALABRAS CLAVES}

Evaluación investigativa, pedagogía de la educación superior, asociación teórico práctica, calidad educativa, nivel de competitividad, memorización, proceso, sistema de aprendizaje, construcción red conceptual.

A nivel universitario se cumple una labor educacional muy importante y compleja que nos permite al igual que en las labores educacionales a nivel secundario o de la básica primaria establecer y crear nuevos enfoques y teorías al interior de ambientes que como los de tas ciencias son imprescindibles en todo programa.

Las ciencias se presentan bajo multiplicidad de programas de estudio a todo nivel universitario secundario y de la básica, pero nos preguntamos ¿es posible que a nivel universitario las acciones educativas en ciencias nos permitan establecer nuevas teorías, metodologías y enfoques? y ¿cómo éstas se concretan y encuentran presentes en los diferentes programas universitarios?

La acción educacional universitaria requiere de marcos conceptuales ganados a través de las teorías educacionales y configurados en una acción pedagógica y docente, sin embargo estos esquemas se han presentado en forma latente y sus cambios presentan grandes dificultades por la complejidad de sus estructuras: por lo anterior se hace necesario establecer en secuencias, nuevas acciones que permitan ir creando diferentes tipos de dinámicas y a la vez nuevas teorías y metodologías.

\footnotetext{
* Msc Biología, profesor titular Biologia, Departamento de Biología. Universidad Pedagógica Nacional

* MGT Física, profesora catedrática titular Departamento de Biología, Universidad Pedagógica Nacional. 
Uno de los campos más preocupantes de las teorías educacionales es la evaluación, por sus concepciones y roles que se le ha impreso entre otros tenemos:

La evaluación en esta primera mitad de siglo $X X$ se refiere a supervigilar la práctica de los exámenes o interrogatorios para comprobar los resultados de la enseñanza" (Murcia) ${ }^{1}$.

Entre 1950-1970 la evaluación presenta un énfasis en el control del rendimiento académico de los estudiantes y su promoción de un nivel a otro"2.

"La evaluación se refiere a la medición del grado en que los objetivos son alcanzados". $(\text { CIDE-CIPCA })^{3}$.

"La evaluación apoyada en principios para estimular la calidad y apoyada en criterios como: a) utilidad, b) factibilidad, c) adecuación, d) precisión, cuatro grupos de criterios seleccionados, jerarquizados por el comité de evaluación de la Universidad de Stanfort y consideran que a su vez se deben estar evaluando" (Stufflebeam) ${ }^{4}$.

“La evaluación como elemento dependiente de los objetivos" (Bloom, Tyler. Popham) 5 .

“La evaluación como medida del conocimiento"6.

Para cada enfoque la evaluación se presenta considerada con nuevos elementos, se denota aislada, así solamente es la respuesta a un cúmulo de contenidos o la respuesta a unos objetivos o a adecuación de un elemento en un proceso, pero es posible que ¿la evaluación pueda lograr una especificidad para el campo de las ciencias donde encontramos más elementos y procesos de interacción?.

Para algunos enfoques que se le ha asignado a la evaluación, también se le ha asociado modelos de acuerdo con las profesiones como en la medicina-evaluación diagnóstica, en la ingeniería, reingeniería (procesos) fortalezas y debilidades, en La comunicación social y artes, evaluación -entrevista-, las cuales se han adoptado a las acciones educacionales unas con buenos resultados, otras no muy buenos, pero nos preguntamos: ¿cuál podría ser uno de los mejores enfoques para la formación de licenciados?

Uno de los posibles caminos establecidos por nosotros en una actividad integradora dentro del eje conceptual célula, el componente físico y el componente biológico, esta en la evaluación investigativa como un proceso, permitiéndonos así, implementar cambios en los procesos de enseñanza-aprendizaje, primero: porque la evaluación pasa de ser un elemento para convertirse en un proceso entre el alumno y su acción de saber, segundo: porque deja de lado el estatisismo al estar en el centro y dinamizarse en la periferia.

\footnotetext{
${ }^{1}$ MURCIA FLORIAN, Jorge. Proceso pedagógico y Evaluación. Colección pedagógica S. XXI, Santafé de Bogotá, D.C Colombia, 1991.

2 ibidem , 3. pág. 84.

${ }^{3}$ MARTINIC S. Walker H (editores). El umbral de lo legitimo-evaluación de la acción cultural. CIDE-CIPCA. Santiago de Chile, 1987.

${ }^{4}$ STUFFLEBEAN, D y otros La evaluación Educativa, evidencias científicas y cuestionamientos políticos. Ed. Casa Editorial Bogotá. 1983.

${ }^{5}$ Ibidem., 3, pág. 106.

${ }^{6} \mathrm{lbdem}$. 3. pág. 89 
La evaluación investigativa, es un proceso, no un elemento del aprendizaje, por que establece el dinamismo entre el alumno, los conceptos, los objetivos y sus múltiples desarrollos, permitiendo un mayor interés y un cambio actitudinal y conceptual hacia la consecución del saber A la vez establece la necesidad de integración de saberes para llegar a su concreción y propone nuevas metodologías de trabajo en el aula, entre docentes y estudiantes.

La evaluación investigativa por lo tanto se constituye en la práctica académica a través de la cual se realiza el proceso de búsqueda, la cual debe ser ordenada, coherente que posea unas reglas que impidan que el proceso sea casual y discontinuo.

Este proceso facilita la formación de estudiantes capaces de. -aprender a pensar problemas reales donde hay aplicación a situaciones de la vida cotidiana, por ejemplo: cirrosis hepática como una disfunción del hepatosito, que permite además una formación en valores y actitudes, respecto al alcoholismo. -El manejo de bases de información y consulta bibliográfica, sintetizar la información y sus fuentes, ganando una visión global en contenidos, - a su vez la visión global Le permite profundizar y ampliar contenidos no contemplados en el aula de clase, —produciendo integración y encadenamiento no sólo para un campo, sino para las demás ciencias.

El trabajo en grupo le ayuda a la socialización de su búsqueda aprendiendo a respetar las opiniones y concepciones, creando valores de tolerancia, respeto y convivencia. La sustentación oral como nivel de expresión de sus conceptualizaciones y conclusiones, facilita y prepara la acción de formación de licenciados en el aspecto de la comunicación.

Este proyecto de trabajo se viene implementando nace dos semestres en el departamento de biología con alumnos del segundo semestre en el componente célula, con una población de aproximadamente 28 alumnos El proceso parte de una pregunta problema, como por ejemplo:

En el proceso de a absorción y transformación de la glucosa intervienen diferentes organelos y sustancias ¿Explique qué tipos de organelos intervien, cuál es su acción? y ¿cuál es el resultado energético del proceso?

Se realiza una práctica experimental conjunta de laboratorio que presenta estrecha relación con el problema planteado.

Seguidamente los estudiantes trabajan en grupos de tres y tienen un tiempo de ocho días para solucionarlo, extraaula. Posteriormente se realiza un seminario taller para integración y análisis de los presupuestos teóricos y de la parte experimental.

Los resultados obtenidos mediante este proceso de evaluación investigativa nos permiten puntualizar los siguientes aspectos:

1 Incrementa el interés por La evaluación y el aprendizaje.

2 Ayuda a la integración de diferentes disciplinas.

3. Facilita la flexibilidad de enfocar la respuesta en un problema abierto.

4. Da oportunidad de analizar lo consultado.

5 Favorece la complementación de conceptos que facilitan la comprensión y ampliación de la solución del problema.

6 Desarrolla habilidad en el manejo de material instrumental de laboratorio. 
7. Actualiza la base de datos a través de Los diferentes medios de información.

\section{BIBLIOGRAFÍA}

NIÑO, Libia Stella. Seminario Internacional sobre evaluación institucional, Edgham, Inglaterra, abril de 1989.

STUFFLEBEAN, Daniel y otros. La evaluación educativa. Evidencias científicas y cuestionamientos políticos. Ed. Casa Editorial, Bogotá, 1983.

MURCIA, Jorge. Proceso pedagógico y evaluación. Editorial Collección. Pedagogía S. XXI. Santafé de Bogotá, Colombia. 1991.

SOTO, Rocío y otro. Consideraciones básicas respecto a la tarea universitaria de investigación educativa. Revista de la Educación Superior, México, Vol. 24, $\mathrm{N}^{0}$ 93, ene mar, 1995.

EDWARS, Verónica. "El currículum y la práctica pedagógica, análisis de dos contextos en formación de docentes en Chile", en Revista de la Sociedad Colombiana de Educación, $\mathrm{N}^{0} 31,1995$.

ESCOBEDO, David H. Pensando en la formación de los docentes del tercer milenio, Revista Actualidad Educativa, Bogotá. Ene-feb. 1995.

MONCAYO, Guido A. y otros. Ciencias, Naturaleza y Salud, Educar, SA. Santafé de Bogotá, Colombia, 1998. 